\title{
Analysis on Hawking Radiation and Steady State Universe by Siva's Theories
}

\author{
Siva Prasad Kodukula, ${ }^{1, * *}$ \\ ${ }^{1}$ Adviser- Centre for Innovation \& Discovery, Bheemunipatnam-, India \\ ${ }^{2}$ Employee of Jindal Steel \& Power Ltd, Angul, India \\ *Corresponding author: sivkod@gmail.com
}

Received March 01, 2014; Revised April 08, 2014; Accepted April 11, 2014

\begin{abstract}
Every black hole will have a singularity at its center. The mass ' $M$ ' and Horizon radius ' $R$ ' are inter related by an equation $\mathrm{M}^{3}=2.914 \times 10^{38} \mathrm{R}$. As long as it remains as a stable black hole the mass ' $\mathrm{M}$ ' \& Horizon Radius ' $\mathrm{R}$ ' will remain constant with $\mathrm{M}=6.58 \times 10^{5} \mathrm{Kgs}$ and $\mathrm{R}=9.767 \times 10^{-22} \mathrm{mts}$. This is the hole connecting all the singularities of the universe. These are always constant for all black holes of the universe. Heavy black holes will lose mass through this hole and will be pumped to other locations of universe to keep the density of the matter created in steady state with the expansion of the universe. The mass loss calculated is $4.953083423 \times 10^{5} \mathrm{Kg} / \mathrm{sec}$. The mass loss is almost same as that of life time of black hole in Hawking Radiation. Thus the mass loss of black hole is not through hawking radiation it is through the singularity point of black hole.
\end{abstract}

Keywords: Quantum Gravity, space time fluid, K-Suryon, singularity of black holes, Hawking Radiation, universe

Cite This Article: Siva Prasad Kodukula, "Analysis on Hawking Radiation and Steady State Universe by Siva’s Theories.” International Journal of Physics, vol. 2, no. 2 (2014): 30-33. doi: 10.12691/ijp-2-2-1.

\section{Introduction}

'Siva's Classical Equations for Space Time and Matter' [8] describes how to calculate the space time fluid density for any mass. The equations are

$$
\begin{gathered}
\mathrm{m}=7.06505184 \times 10^{12}\left(\mathrm{~d}^{1 / 3}\right) \\
\gamma \mathrm{d}^{8 / 3}=1.686656885 \times 10^{12}
\end{gathered}
$$

Where ' $\gamma$ ' is 'space time fluid density' of mass ' $m$ '

The same concept has been applied to massive black holes. The complete black hole is a mass spread over a radius and a singularity [10] at its center. 'Virtual KSuryon' $[9,11]$ is a singularity with zero mass. But black hole[10] is a singularity with mass. The difference is space time.

K-Suryon will not have space time. But black hole will have space time and a singularity at its center. It follows equation for singularity and Schwarzschild radius.

\section{Discussion}

We have Schwarzschild radius equation.

$$
\text { Schwarzschildradius }(\mathrm{R})=\frac{2 \mathrm{GM}_{\mathrm{B}}}{\mathrm{c}^{2}}
$$

So we can find the ' $R$ ' for a black hole of mass $M_{B}$, It will increase as the mass increases.

This shows the increment in surface area of Horizon for a black hole with mass. Let us suppose a mass from outside of black hole has been swallowed by a black hole of mass ' $\mathrm{M}_{\mathrm{B}}$ ' so that the mass of black hole has become ' $n$ ' times i.e ' $n M_{B}$ '.

For this ' $\mathrm{M}_{\mathrm{B}}$ ' we can calculate the space time fluid density.

As per equation (1) the radius of space time fluid can be calculated.

If we substitute the ' $\mathrm{nM} \mathrm{B}_{\mathrm{B}}$ ' in place of ' $\mathrm{m}$ ' of equation (1)

$$
\mathrm{nM}_{\mathrm{B}}=7.06505184 \times 10^{12}\left(\mathrm{~d}^{1 / 3}\right)
$$

where ' $\mathrm{d}$ ' is space time fluid radius of newly formed black hole.

$$
\mathrm{d}=\left(\mathrm{nM}_{\mathrm{B}} / 7.06505184 \times 10^{12}\right)^{3}
$$

If we substitute this value of ' $d$ ' in equation (2)

$\gamma\left[\left(\mathrm{nM}_{\mathrm{B}} / 7.06505184 \times 10^{12}\right)^{3}\right]^{8 / 3}=1.686656885 \times 10^{12}$

Therefore

$$
\begin{gathered}
\gamma=\frac{1.686656885 \times 10^{12} \times(7.06505184)^{8} \times 10^{96}}{\left(\mathrm{nM}_{\mathrm{B}}\right)^{8}} \\
=1.047006954 \times 10^{115 / \mathrm{n}^{8} \mathrm{M}_{\mathrm{B}}{ }^{8}} \\
\therefore \gamma=\frac{1.05 \times 10^{115}}{\mathrm{n}^{8}\left(\mathrm{M}_{\mathrm{B}}\right)^{8}}
\end{gathered}
$$

Density of new black hole can be calculated as-

$$
\text { Mass }=\mathrm{nM}_{\mathrm{B}}
$$


Radius is ' $R$ ' and volume $=(4 / 3) \pi R^{3}$

$$
\text { Density }=\frac{n M_{B}}{(4 / 3) \pi R^{3}}
$$

Equation (5) can be equated with equation (6)

Therefore

$$
\frac{\mathrm{nM}_{\mathrm{B}}}{(4 / 3) \pi \mathrm{R}^{3}}=\frac{1.05 \times 10^{115}}{\mathrm{n}^{8}\left(\mathrm{M}_{\mathrm{B}}\right)^{8}}
$$

Therefore

$$
\left(\mathrm{nM}_{\mathrm{B}}\right)^{9}=2.474 \times 10^{115} \mathrm{R}^{3}
$$

We can say

$$
\mathrm{M}_{\mathrm{O}}{ }^{3}=2.914 \times 10^{38} \mathrm{R}
$$

where ' $\mathrm{M}_{\mathrm{O}}$ ' is mass of newly formed black hole with radius ' $R$ '.

We have the relation $\mathrm{R}=\frac{2 \mathrm{GM}_{\mathrm{O}}}{\mathrm{c}^{2}}$.

We have $\mathrm{G}=6.67 \times 10^{-11}, \mathrm{C}=2.997925 \times 10^{8} \mathrm{mt} / \mathrm{sec}$.

Therefore

$$
\begin{aligned}
& \text { Mo }=6.58 \times 10^{5} \mathrm{Kgs} \\
& \mathrm{R}=9.767 \times 10^{-22} \mathrm{mt}
\end{aligned}
$$

Thus we can say that the mass ' $M$ ' and Horizon radius ' $\mathrm{R}$ ' of a black hole is inter related by an equation $\mathrm{M}^{3}=$ $2.914 \times 10^{38} \mathrm{R}$. Thus all the stable black holes with mass ' $M$ ' \& Horizon Radius ' $R$ ' will remain constant with $M=$ $6.58 \times 10^{5} \mathrm{Kgs}$ and $\mathrm{R}=9.767 \times 10^{-22} \mathrm{mts}$. This is the hole connecting all the singularities of the universe. These are always constant for all black holes of the universe.

If this is the case, when a star becomes a black hole, the mass and the Schwarzschild radius of that black hole will be higher than the above calculated constant parameters i.e $\mathrm{M}=6.58 \times 10^{5} \mathrm{Kgs}$ and $\mathrm{R}=9.767 \times 10^{-22} \mathrm{mts}$. So it is understood that the black hole will not be stable and it will loose its mass through the hole surrounded by its singularity point existed at its center. This is the hole connecting all the singularities of the universe.

This mass will be redistributed to the universe since it requires continuous creation of matter to maintain in steady state with expansion.

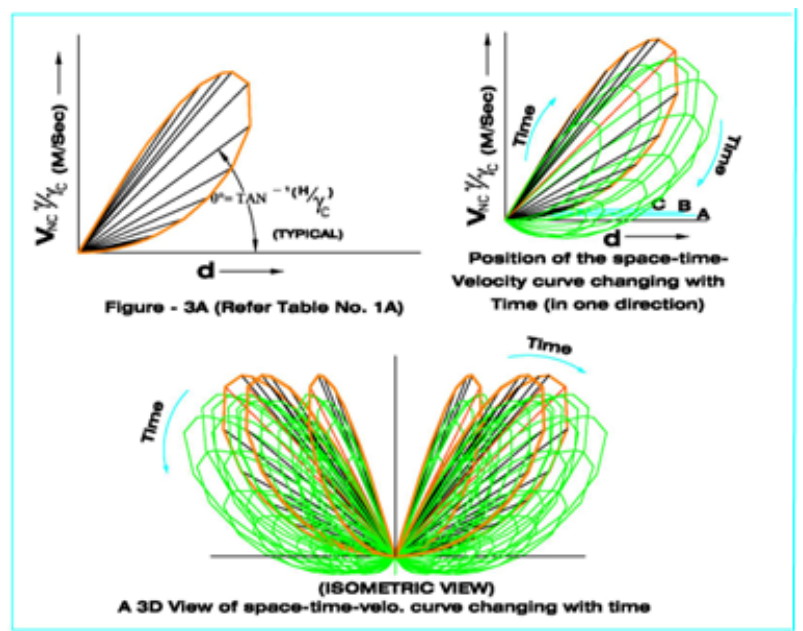

FILMS OF THE UNIVERSE References [5][6]

Figure 1.
As per the "Heart of God Model'[6] of the universe, the universe is like human heart pumps the matter in to this universe for every $7.6813002 \times 10^{-44}$ seconds which is the duration of film change of the universe as per 'Film theory of the Universe' [5,6]. Figure 1 shows the graphical representation of films in this universe. The pumping of new matter is a continuous process in this universe.. The density of the matter will be constant. The creation of matter will be compensated by expansion so as to maintain the density of matter of the universe even though it is expanding. This is similar result of Sir Fred Hoyle's steady state theory.

Thus the density of Mass draining per second through singularity point of black hole $=$ the density of the mass pumped out of 'Heart of God' per second.

\subsection{Calculation for Loss of Mass of Black Hole}

Calculation for the loss of density of mass for the black hole per second through its own Singularity:

We have density of Black hole $=\frac{M}{(4 / 3) \pi R^{3}}$.

$$
\begin{aligned}
& \text { And } \mathrm{R}=\frac{2 \mathrm{GM}_{\mathrm{O}}}{\mathrm{c}^{2}} \\
& \begin{aligned}
\text { Density }= & \frac{\mathrm{M}}{(4 / 3) \pi \mathrm{R}^{3}}=\frac{\mathrm{M}}{(4 / 3) \pi\left(2 \mathrm{GM} / \mathrm{c}^{2}\right)^{3}} \\
& \therefore \text { Density }=\frac{3 \mathrm{c}^{6}}{32 \pi \mathrm{G}^{3} \mathrm{M}^{2}}
\end{aligned}
\end{aligned}
$$

This singularity will be connected to all the singularities of the universe including 'K-Suryon', the basic building block of matter.

As per the concept, the mass lost through its singularity = the mass pumped out by 'Heart of the God model'.

Already 'Heart of God model' Explained and calculated the density of the mass creation in one second.

\subsection{Review of the Calculation by 'Heart of God Model'[6]}

According to film theory, universe is made up of films which are changing for every $7.6813002 \times 10^{-44} \mathrm{sec}$. The events are occurring due to change of film. It is like a movie in which sixteen films are changing at every second. In 'Double Relativity' it is described that a force will act according to equation $\mathrm{V}=\mathrm{Hd}$ in one direction and $\mathrm{Vd}=\mathrm{K}$ in opposite direction. Even though time is a quantized quantity the space-time is not. The space-time is a continuous one. So how to make this in to one system which obeys continuous expansion of space with time. So there must exist something in our conventional fourdimensional world, which we have described as nothing in full truth. Already we have described that light is that nothing which seems like photon in this four-dimensional space-time. The same thing will exist between two films. This is a singularity concept of 'K-suryon' explains the creation of mass from this singularity. So the process of continuous creation of matter exists in between two films of the universe.

\subsection{Calculation for Quantity of Mater Creates during Film Change [6]}


Let us suppose, for the first film, space of the universe is with diameter ' $\mathrm{d}_{\mathrm{o}}$ ' and the matter is existed with velocity ' $\mathrm{V}_{\mathrm{o}}$ '. It can be related with the equation $\mathrm{V}_{\mathrm{o}}=\mathrm{H} \mathrm{d}_{\mathrm{o}}$. As per Double relativity Effect the $V_{\min }=1000 \mathrm{mts} / \mathrm{sec}$. Since it is first film.

Therefore it's diameter ' $\mathrm{d}_{\mathrm{o}}$ ' $=\mathrm{V}_{\min } / \mathrm{H}=$ $1.9147644 \times 10^{22} \mathrm{mts}$. Where ' $\mathrm{H}$ ' is Hubble's constant i.e equal to $5.223 \times 10^{-20} / \mathrm{sec}$ as per 'derivation of Siva's Constant ' $\mathrm{K}$ '[7].

\subsubsection{Data For the First Film}

Dia of the space ' $\mathrm{d}_{\mathrm{o}}$ '

$$
=\frac{\mathrm{V}_{\min }}{\mathrm{H}}=\frac{1000}{5.2225746 \times 10^{-20}}=1.9147644 \times 10^{22} \mathrm{mt}
$$

Velocity of matter $=1000 \mathrm{mts} / \mathrm{sec}$

Time taken for film change[5] i.e 't' $=7.6813002 \times 10^{-44}$ sec.

So with in this time the matter at a point at ' $\mathrm{d}_{\mathrm{o}}$ ' dia will move to a dia of 'd' i.e. dia of second film.

So we can calculate change of dia during film change.

\subsubsection{Data for the Second Film}

Change of dia of space 'd' from first film to second film

$=\mathrm{V}_{\min } \times \mathrm{t}$

$=1000 \times 7.6813002 \times 10^{-44} \mathrm{mts}$.

$=7.6813002 \times 10^{-41} \mathrm{mts}$.

Change of volume for a change of film

$\therefore=(4 / 3) \pi(\mathrm{d} / 2)^{3}=2.373027865 \times 10^{-121}$

Let us keep the density of matter constant and compensate the expansion.

For

one film (the first film) mass of matter

$=$ Mass of whole universe

$=6.448233042 \times 10^{48} \mathrm{kgs}$

(As calculated in 'Double Relativity Effect') [5].

The density of matter for the first film

$$
\begin{aligned}
& =\frac{\text { Mass of the universe }(\mathrm{M})}{\text { Volume of space occupied (Volume) }} \\
& =1.7542685 \times 10^{-18} \mathrm{~kg} / \mathrm{m}^{3}
\end{aligned}
$$

For second film,

the created matter

$=$ Same as first filmChange of volume from first film to second

= Volume

$=2.373027865 \times 10^{-121}$

(As per above calculation)

Created matter during film change

=Volume'density

$=2.373027865 \times 10^{-121} \times 1.7542685 \times 10^{-18}$

$=4.162928033 \times 10^{-139}$

Duration of film change $[5,6]$ ' $\mathrm{t}$ ' $=7.6813002 \times 10^{-44} \mathrm{sec}$
The observable matter distribution

between first film and second film

Created matter or mass $(\mathrm{M})$ during film change

Change of volume of space during film change

$=1.7542685 \times 10^{-18} \mathrm{Kg} / \mathrm{m}^{3}$ per film

$=2.28381713 \times 10^{25} \mathrm{Kg} / \mathrm{m}^{3}$ per sec

Here the diameter of the universe ' $d$ '

$=\mathrm{V} / \mathrm{H}$

$=1.9147644 \times 10^{22} \mathrm{mts}$.

This is constant. It will not change with film change. For first film, second film it is same

Therefore finally the density of the matter created while film change, can be calculated by distributing the created matter throughout its constant volume

The density of the continuous creation of matter

$$
\begin{aligned}
& =\left(2.28381713 \times 10^{25} /(4 / 3) \pi\left(1.9147644 \times 10^{22} / 2\right)^{3} \mathrm{~kg} \mathrm{~m}^{-3} \mathrm{sec}^{-1}\right. \\
& =\left(2.28381713 \times 10^{25}\right) / 3.6757389 \times 10^{66} \mathrm{~kg} \mathrm{~m}^{-3} \mathrm{sec}^{-1} \\
& =6.21321914 \times 10^{-42} \mathrm{~kg} \mathrm{~m}^{-3} \mathrm{sec}^{-1}
\end{aligned}
$$

This is similar result as predicted by 'steady state theory' which states that universe is always existed in a steady state, the expansion of the universe is compensated by the continuous creation of matter which is viewed as a property of space, and that despite local evolutionary process, the universe as a whole is not evolving. The rate at which matter would have to be spontaneously created to compensate for the universe's expansion (about $10^{-43} \mathrm{~g} \mathrm{~cm}^{-3} \mathrm{sec}^{-1}$ ) [13].

As calculated above the density of mass Created during film change of the universe $=2.28381713 \times 10^{25} \mathrm{Kg} / \mathrm{m}^{3}$

As per 'Film Theory of the Universe' [5] no. of Films per second $=1 / 7.6813002 \times 10^{-44}$.

Therefore total density of matter created or pumped out

$$
\text { per second }=\frac{2.28381713 \times 10^{25}}{7.6813002 \times 10^{-44}}
$$

This is quantitatively equal to equation (9)

$$
\begin{aligned}
& \frac{3 c^{6}}{32 \pi \mathrm{G}^{3} \mathrm{M}^{2}}=\frac{2.28381713 \times 10^{25}}{7.6813002 \times 10^{-44}} \\
\therefore \quad \mathrm{M}^{2}= & \left(3 \mathrm{c}^{6} \times 7.6813002 \times 10^{-44}\right) /\left[\begin{array}{l}
32 \pi\left(6.672 \times 10^{-11}\right)^{3} \\
\times 2.28381713 \times 10^{25}
\end{array}\right] \\
= & 2.45330354 \times 10^{11} \\
\therefore \mathrm{M}= & 4.953083423 \times 10^{5} \mathrm{Kg} .
\end{aligned}
$$

So we can conclude the mass loss $(\mathrm{M})$ of black hole per second $=4.953083423 \times 10^{5} \mathrm{Kg}$.

Thus we can conclude that the mass of a black hole will be drained out through a hole which connects all the singularities or the points from the matter pumped out by 'Heart of the God'[6].

The density of the matter drained out per second is equal to the density of matter created per second.

Thus we reached a conclusion that the mass of black hole will be drained out through its singularity as a mass loss.

Similar concept of mass loss has been calculated by black hole physics in terms of 'Hawking Radiation' [3]. 


\subsubsection{Hawking Radiation in Terms of Mass Loss}

Life time of a black hole $[1]=5120 \pi \mathrm{G}^{2} \mathrm{M}^{3} / \mathrm{hc}^{4}$

$$
\begin{aligned}
\therefore \quad \mathrm{M}^{3} & =\mathrm{hc}^{4} / 5120 \pi \mathrm{G}^{2} \\
& =7.474930 \times 10^{16}
\end{aligned}
$$

Mass loss per second $=4.212459223 \times 10^{5} \mathrm{Kg}$.

This is almost equal to the mass loss of black hole to pump out the matter by 'Heart of God' to compensate expansion of the universe as well as to keep the universe in steady state i.e. $4.953083423 \times 10^{5} \mathrm{Kg}$.

- This analysis elaborates the process of matter creation as explained in steady state theory or 'Heart of the God model' to compensate the expansion of universe.

- The matter absorbed by the black holes will be spread over the universe in some other area.

- The concept of 'Film theory of Universe' 'Heart of the God model' is in line with the present day calculations of black hole physics and black hole thermodynamics.

- The calculations and the idea of mass loss of black hole are correct but not as expected as Hawking Radiation. The mass loss is through the hole surrounded by singularity existed at its center and further will be divided in to K-Suryons to pump out as a continuous creation of matter to keep the universe in steady state.

- The rate of mass loss of black holes i.e $4.953083423 \times 10^{5} \mathrm{Kg} / \mathrm{sec}$ can be observed by 'molecular gas kinematics'[12].

\section{Conclusions}

1. The mass ' $M$ ' and Horizon radius ' $R$ ' are inter related by an equation $\mathrm{M}^{3}=2.914 \times 10^{38} \mathrm{R}$.

2. The mass ' $M$ ' and Horizon radius ' $R$ ' of all black holes are same $M=6.58 \times 10^{5} \mathrm{Kgs}$ and $\mathrm{R}=9.767 \times 10^{-22} \mathrm{mts}$.

3. Black hole with above parameters is the hole connecting all the singularities of the universe.

4. All the black holes which are greater in mass will loose its mass through its singularity point at a rate $4.953083423 \times 10^{5} \mathrm{Kg} / \mathrm{sec}$.

5. The mass lost through this singularity point will compensate the creation of rate of density of matter pumped out by 'Heart of the God' so that the expansion of the universe compensate with creation of matter. And the mass loss is almost equal to the mass loss of black hole calculated by life time calculation of 'Hawking Radiation'.
6. Hawking radiation expecting the mass loss from the black hole as radiation (or similar explanations like particle pair in quantum black holes $[2,3,4]$ ) which is not supported by the analysis.

7. The rate of mass loss from the black hole can be observed by 'molecular gas kinematics' [12].

\section{References}

[1] Adam R. Brown, "Tensile Strength and the Mining of Black Holes” Phys. Rev. Lett. 111, 211301 (2013).

http://arxiv.org/abs/1207.3342. [Accessed March. 1, 2014].

[2] George, F R Ellis. "Astrophysical black holes may radiate but they do not evaporate." arXiv: 1310.4771v2 [gr-qc] 20 Oct 2013 Available: http://arxiv.org/pdf/1310.4771.pdf. [Accessed March. 2, 2014].

[3] Hawking,S.W., "Particle creation by Black holes". commun. 43, 199 -220 (1975) maths.phys. Available: http://arxiv.org/pdf/hep-th/9203052.pdf. [Accessed March. 2, 2014].

[4] Hawking, S.W. \& Penrose. The Nature of space and Time, Princeton University press, pp. 38-60, 1998.

[5] Kodukula, S.P., Double Relativity Effect \&Film Theory of the Universe. Raleigh,NorthCorolina:Lulu.com, pp 13-32, 50, 2009.

[6] Kodukula S.P,Heart of the God with Grand proof Equation - A classical approach to quantum theory. Raleigh, NorthCorolina:Lulu.com, pp3-9, 53, 2009.

[7] Kodukula, S.P, "Derivation of Siva's Constant of Physics", International Journal of Advancements in Research \& Technology', 2(1), Jan. 2013. Available: http://www.ijoart.org/docs/Derivation-of-Sivas-Constant-K-ofPhysics.pdf. [Accessed March. 1, 2014].

[8] Kodukula, S.P, "Siva's Classical Equation for Space Time and Matter", International Journal of Advancements in Research \& Technology', 2(8), Aug. 2013. Available: http://www.ijoart.org/docs/Sivas-Classical-Equation-for-SpaceTime-and-Matter.pdf. [Accessed March. 1, 2014].

[9] Kodukula. S.P, "Siva's Theory of Quantum Gravity". American Journal of Modern Physics, 3(1), 16-19. Jan. 2014. [Accessed March. 1, 2014].

[10] Kodukula,S.P,"Siva's Equation for Singularity of Black Holes”. International Journal of Astrophysics and Space Science,1(4),1619. 2013.

[11] Kodukula, S.P, "New Discovery about Prediction of a Particle 'KSuryon’ as Basic Building Block of Mass”. International Journal of physics, 2(1), 12-14. 2013,

[12] Timothy A. Davis et al., "A black-hole mass measurement from molecular gas kinematics in NGC4526" arXiv:1301.7184v1 [astro-ph.CO] 30 Jan 2013 Available: http://arxiv.org/pdf/1301.7184.pdf [Accessed March. 2, 2014].

[13] H. Bondi \& T. Gold, "The Steady State Theory of the Expanding Universe" Monthly Notices of Royal Astronomical Society, Vol.108, p.265 (1948). Available: http://adsabs.harvard.edu/full/1948MNRAS.108..25B [Accessed April 5,2014]. 